\title{
Publisher Correction: A new foe in folate metabolism
}

Zhengwei Wu and Wai Leong Tam (1)

Correction to: Nature Metabolism https://doi.org/10.1038/s42255-021-00474-9, published online 18 November 2021.

In the version of this article initially published, a versioning error during composition led to mistakes in the order and completeness of the author list and in-text citations of ref. 3. The errors have been corrected in the online version of the article.

Published online: 13 December 2021

https://doi.org/10.1038/s42255-021-00513-5

๑) Springer Nature Limited 2021 